Conclusions Recommended antibiotic treatment was nearly always offered to patients. However general advice and written documentation were poor and non-attendance at follow up was high. Urine culture testing and documentation should be significantly improved for initial management, and a robust system developed to assess compliance with treatment, $\mathrm{PN}$ and symptom resolution.

\section{P2.027 GENEXPERT GBS AND CT/NG REAL TIME PCR ASSAYS AS INNOVATIVE TOOLS FOR CERVICO-VAGINAL INFECTIONS'SCREENING}

doi:10.1136/sextrans-2013-051184.0292

G Di Renzo, G Babucci, I Giardina, M Cimino, E Picchiassi, F Tarquini, M Centra, G Coata. University of Perugia, Perugia, Italy

Background Group B Streptococcus (GBS), Chlamydia trachomatis (CT) and Neisseria gonorrhoeae (NG) cervico-vaginal infections can be involved in pregnancy complications such as preterm birth and premature rupture of membrane (PROM). These infections can also be transmitted to the newborn during delivery leading to serious consequences. Therefore, CDC guidelines suggest microbial prenatal screening for administration of target prophylaxis based on culture results. To accurately predict the colonisation of genital tract, the test should be better performed during labour, because microbial presence may be transient/intermittent and re-colonisation can occur. GeneXpert ${ }^{\circledR} G B S$ and GeneXpert ${ }^{\circledR} \mathrm{CT} / \mathrm{NG}$ tests (Cepheid), fully-automated, easy-to-use and rapid PCR-assays (about 45 and $90 \mathrm{~min}$, respectively) can be the right alternative to culture tests (at least 72 hours).

This study evaluates the advantages of GeneXpert ${ }^{\circledR} G B S$ in the management of women, with unknown cervico-vaginal microbial status, during labour. Moreover, it assesses whether the prevalence of CT, NG and GBS infections is higher in pregnancy complicated by preterm labour or PROM.

Methods During a four months' period, all women with singleton pregnancy at beginning of labour either-term or preterm or PROM were enrolled. Exclusion criteria were planned caesarean section or recent use of systemic or topical antibiotics. Cervico-vaginal (for $\mathrm{CT} / \mathrm{NG}$ ) and vaginal-rectal (for GBS) swabs were collected from each patient and analysed by GeneXpert ${ }^{\circledR} \mathrm{GBS}$ and GeneXpert ${ }^{\circledR} \mathrm{CT} /$ NG assays on GeneXpert ${ }^{\circledR}$ System.

Results CT/NG screening showed positive results only among PROM pregnancies (2.5\% CT positive) while no positive results were found among preterm/term pregnancies.

Among pregnant women analysed for GBS, $24.4 \%$ resulted positive and $75.6 \%$ negative. Only positive patients received IAP, instead of current guidelines, for which all patients would have been treated due to unknown GBS infection status.

Conclusion With GeneXpert ${ }^{\circledR}$ GBS test, we could correctly manage all women and reduce administration of IAP. We calculated that the savings for the hospital was 3,500 EUR every three months.

\section{P2.028 EVALUATION OF THE VAGINAL MICROBIOTA USING QUANTITATIVE REAL-TIME PCR}

doi:10.1136/sextrans-2013-051184.0293

K Shalepo, V Nazarova, J Menukhova, A Savicheva. Ott Institute of Obstetrics and Gynecology Academy of Medical Sciences of Russian Federation, St Petersburg, Russian Federation

Background For characterization of the vaginal microflora, it is often necessary to assess quantities of microorganisms. The study objective was to evaluate vaginal microflora in norm, bacterial vaginosis (BV) and vulvovaginitis (VV) using quantitative real-time PCR. Methods A total of 255 women of reproductive age, who addressed a gynaecologist due to vaginal discharge, were included in the study. BV was diagnosed using the Amsel criteria and the Nugent score. VV was diagnosed on the basis of clinical presentation and microscopy results. The patients were divided into 3 groups: healthy $(\mathrm{n}=128), \mathrm{BV}(\mathrm{n}=48)$ and VV $(\mathrm{n}=79)$. Vaginal swabs were analysed using a real-time PCR test (Femoflor, DNATechnology, Russia), which assesses total bacteria, lactobacilli, anaerobic bacteria and genital mycoplasmas. The quantities of the microorganisms were summarised as median values in each group of patients.

Results In the group of healthy women, total bacteria were present in a median load of $7.9 \times 10^{6}$ DNA copies per reaction, in BV group - $3 \times 10^{7}$, in VV group - $6.7 \times 10^{6}$. The median loads of lactobacilli in the group of healthy women, BV and VV groups were $4 \times 10^{7}$, $1.4 \times 10^{4}$ and $1.6 \times 10^{6}$ copies per reaction, respectively. Quantities of Gardnerella vaginalis/Prevotella bivia/Porphiromonas spp. in the three groups were $2.5 \times 10^{2}, 8.7 \times 10^{6}$ and $5.8 \times 10^{2}$ copies per reaction, respectively. Megasphaera spp./Veillonella spp./Dialister spp were present in the three groups in loads of $1,4.3 \times 10^{5}$ and $5.8 \times 10^{1}$ copies per reaction, respectively. Atopobium vaginae was found in the three groups in quantities of $1,2.1 \times 10^{6}$ and 1 copies per reaction, respectively. The median values of Mycoplasma hominis were equal in the three groups (median values 1 copy per reaction). The quantities of Ureaplasma urealyticum/parvum were $1.4 .9 \times 10^{2}$ and 1 copies per reaction, respectively.

Conclusion Real-time PCR is a fast and accurate tool for the assessment of the vaginal microbiota.

\section{P2.029 ALL THAT IS GENITAL IS NOT VENEREAL}

doi:10.1136/sextrans-2013-051184.0294

S F Kazi, K Godse, S Patil, N Nadkarni. P.D.D.Y. Patil Hospital, Navi Mumbai, India

\section{ALL THAT IS GENITAL IS NOT VENEREAL}

Unusual cases with lesions on genitals

Genital disease (GD) has a broad spectrum. Though sexual transmission obviously plays a major role in its pathogenesis, many diseases with genital presentation are not sexually transmitted diseases (STD) but due to strong clinical resemblance cause a lot of diagnostic confusion and therapeutic misadventures. We report 4 such cases.

Case 1 and 2 A 35 yr old female presented with recurrent attacks of itchy, painful lesions over the genitals along with musculo-articular symptoms. On examination there were classical "knife cut ulcers" over the labia, perianal areas and inframammary folds.

Another 26 year old unmarried female presented with vulvar lesions. Indurated tender swelling, ulceration of the vulva, violaceous plaques with edoema were present.

Histopathology showed epitheloid cell granuloma with occasional Langhans giant cells. Clinicopathological correlation clinched both cases as Metastatic Crohn's disease which responded to a course of oral metronidazole and corticosteroids.

Case 3 A young male presented with penile swelling, multiple discharging ulcers since 2 months. There was distortion of the shape and multiple shallow necrotic ulcers on the shaft of the penis with phimosis. Non- tender right inguinal lymphadenopthy. Biopsy showed multiple tuberculoid granulomas. Mantoux test was strongly positive with high ESR suggesting Genital Tuberculosis. The patient was started on AKT. 3 weeks later ulcers healed with destruction of overlying skin.

Case 4 A 56 year old married male came with raised lesions and a glazed whitish discolouration of glans penis with phimosis and thinning of urinary stream.

Biopsy revealed atypical undifferentiated cells and horn pearls Imprint cytology and FNAC were done and a final diagnosis of Squamous Cell Carcinoma was made which required partial amputation of penis. 